\title{
Attentional coding of categorical relations in scene perception: Evidence from the flicker paradigm
}

\author{
LUKE J. ROSIELLE, BRIAN T. CRABB, and ERIC E. COOPER \\ Iowa State University, Ames, Iowa
}

\begin{abstract}
The purpose of the present investigation was to determine whether the positions of objects in a scene are coded relative to one another categorically (i.e., above, below, or side of; Experiment 1) and to determine whether spatial position in scene perception is coded preattentively or only under focused attention (Experiment 2). In Experiment 1, participants viewed alternating versions of a scene in which one of the objects in the scene changed its categorical relationship to the closest object in the scene, changed only its metric relationship to the closest object in a scene, or appeared and disappeared. Participants were faster at detecting changes that disrupted categoricalrelations than at detecting changes that disrupted only metric relations. In Experiment 2, this categorical advantage still occurred even when participants were cued to the location of the change. These results suggest that categorical spatial relations are being coded in scene perception and that attention is required in order to encode spatial relations.
\end{abstract}

When one briefly views a scene, one gets the qualitative impression that all of the information from that scene is encoded into memory (Henderson \& Hollingworth, 1999b). However, recent research has shown that very little information from the scene is actually coded into working memory (Carlson-Radvansky \& Irwin, 1995; Irwin \& Andrews, 1996; Rensink, O’Regan, \& Clark, 1997, 2000). Because of this lack of complete coding, changes to scenes are sometimes very difficult to detect. For example, Rensink et al. (1997) demonstrated a phenomenon known as change blindness, in which participants, when shown alternating versions of a scene interspersed with blank intervals (the flicker paradigm), failed to quickly detect large changes (e.g., changes in an object's color or position) between the two versions of the scene. Others have shown that changes across cuts in a movie (Levin \& Simons, 1997), changes that occur across saccades (Carlson-Radvansky \& Irwin, 1995; Henderson \& Hollingworth, 1999b; Irwin \& Andrews, 1996), and changes in exemplar-level objects learned on the basic level (Archambault, O'Donnell, \& Schynes, 1999) also induce change blindness (see Henderson \& Hollingworth, 1999a, and Rensink, 2000, for recent reviews).

Several mechanisms have been proposed to explain why changes in scenes are often difficult to detect. For exam-

Portions of this paper were presented at the meeting of the Psychonomic Society, Los Angeles, November 1999. The authors thank Eric Esser and Shala Wyant for their help in conducting the research described in this paper and Veronica J. Dark, Brian E. Brooks, and Lori D. Lindley for their careful reading of an earlier version of this manuscript. Correspondence should be addressed to L. J. Rosielle, who is now at Department of Psychology, P. O. Box 10048, Louisiana Tech University, Ruston, LA 71272 (e-mail: 1rosielle@juno.com). ple, Henderson and Hollingworth (1999b) argue that when people visually scan scenes, a memory representation is formed around the local region of the scene that is currently in foveal vision. Detecting a change involves maintaining gaze at the region of change or directing one's eyes toward the region of change prior to the change taking place, encoding that region in memory, and then redirecting the eyes back to that same region after the change has taken place. Similarly, Rensink (2000) has proposed that focused attention stabilizes the input from the currently attended region of a scene long enough for a change to be detected. Thus, without attention, change detection is unlikely.

Both views posit that when viewing a scene, a detailed representation is formed only of the local region of the scene on which one is currently focusing attention. However, little is currently known about the nature of this representation and what type of information people extract from the representation in order to be able to detect changes.

\section{Coding Position in Scene Perception}

A scene can be thought of as consisting of two fundamental types of visual elements: a set of objects and the spatial positions of these objects. Although numerous studies have investigated what type of information people extract from objects in the context of a scene (see Henderson \& Hollingworth, 1999a, for a review), relatively few studies have investigated how the positions of objects are coded in scene perception.

There are a number of possible ways in which the positions of objects in a scene might be coded. One possibility, suggested by the literature on object recognition (e.g., Biederman, 1987; Hummel \& Biederman, 1992; Hummel \& Stankiewicz, 1996), is that the positions of objects in a 
scene might be coded relative to other objects in the scene using the broad categorical descriptors above, below, and side of. According to this view, the picture frame in Figure 1 might be coded as simply being above the couch, whereas the end table might be coded as being to the side of the couch. Although many possible types of categorical relations might be coded between the objects in a scene (e.g., in front of or behind), coding above, below, and side of would allow the representation to become invariant to many types of changes in an observer's viewpoint. For example, if a viewer were to approach the couch in Figure 1, the picture frame would still be above the couch, allowing for an easy match in memory between the original view of the scene and the new view of the scene.

Several authors have suggested that categorical relations may play a role in individual object recognition (e.g., Biederman, 1987; Cooper \& Wojan, 2000; Hummel \& Biederman, 1992; Hummel \& Stankiewicz, 1996; Humphreys \& Riddoch, 1995; Rosielle \& Cooper, 2001), and it is possible that categorical relations may play a role in scene perception as well. However, many have argued that spatial relations within an individual object may be coded differently than spatial relations between multiple objects in a scene (Epstein \& Kanwisher, 1998; Humphreys \& Riddoch, 1994, 1995; Saiki \& Hummel, 1998a, 1998b), but the exact differences between within-object coding of spatial relations and between-object coding of spatial relations have yet to be specified (but see Simons \& Wang, 1998; Wang \& Simons, 1999).
In contrast to categorical coding, it is possible that only the exact metric positions (e.g., specified in retinal coordinates) of the objects in a scene may be coded in memory (see, e.g., Bülthoff \& Edelman, 1992; Tarr \& Pinker, 1990, 1991; Ullman, 1989, 1996). According to this view, the picture frame in Figure 1 might be coded as being 3 units above and 4 units to the right of a point on the retina. Coding position only metrically may be advantageous for such tasks as navigating through a scene, but whether such a precise representation is generally useful for the purposes of everyday vision is an open question and a subject of some debate in the literature (see Biederman \& Gerhardstein, 1995; Tarr \& Bülthoff, 1995).

It is important to note that categorical coding and metric coding are not mutually exclusive. In fact, some models of object recognition posit that both categorical information and metric information are coded in visual object perception (e.g., Hummel \& Stankiewicz, 1998), and it seems possible that both may be coded in scene perception as well. Thus, the purpose of the experiments reported here was to test whether categorical relations are being coded in scene perception (regardless of whether metric coding occurs) or whether no categorical coding occurs in scene perception at all (and only metric coding occurs).

\section{EXPERIMENT 1}

In Experiment 1, the change blindness phenomenon was used to examine whether the positions of objects in a scene

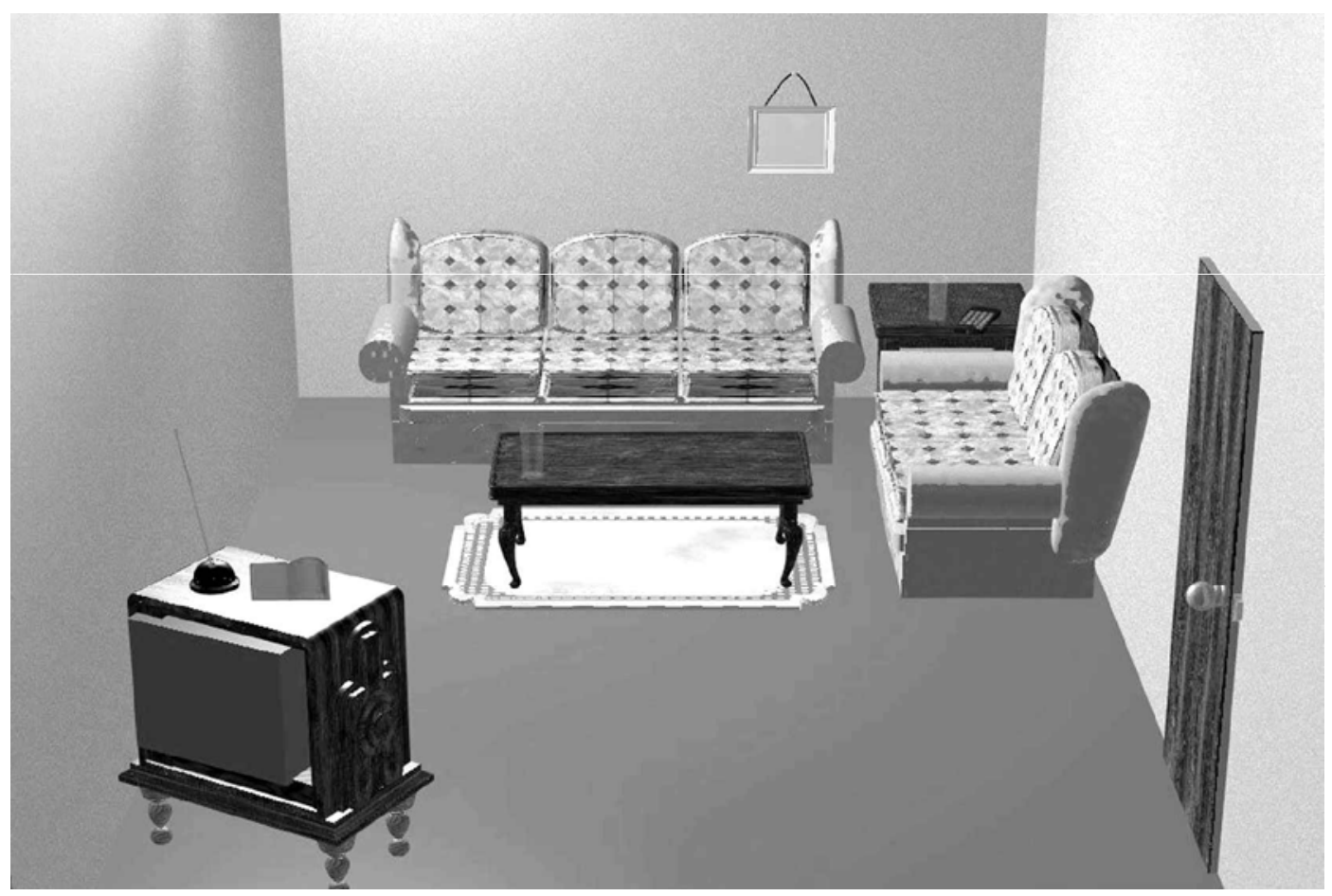

Figure 1. An example of a scene. If above, below, and side of are being coded in scene perception, then the picture frame might, for example, simply be coded as being above the couch. 
are coded categorically or exclusively metrically. The question was, what types of changes in the positions of objects in a scene do people find relatively difficult and relatively easy to detect? In Experiment 1, we assessed whether people are more sensitive to changes in the categorical relations above, below, or side of among the objects in a scene than to equivalent changes in only the metric relations among the objects in a scene. A version of Rensink et al.'s (1997) flicker paradigm was used in which alternating versions of a scene were presented. Between the two versions, one of the objects changed its categorical relationship to the closest other object in the scene (e.g., went from above an object to side of an object), changed only its metric relations to the closest object in the scene (e.g., alternated between two locations both above an object), or appeared and disappeared (Figure 2). The participants' task was to press a button when they detected the object that was changing and to report the identity of that object to the experimenter.

If the categorical relations above, below, and side of are coded in scene perception, then participants should be faster at detecting a change in the position of an object in a scene that disrupts its categorical relationship to the closest object in a scene (categorical change condition) than at detecting a change that disrupts only its metric re- lationship (metric change condition). This advantage for detecting categorical changes should occur even though the absolute distance the object moves is equal in both cases. If, however, only metric coding is used in scene perception, then both the metric changes and the categorical changes should be detected equally fast.

The presence/absence scenes were included in Experiment 1 as an anchor. Rensink et al. (1997) found that presence/absence changes were detected more quickly than were location changes (when the target object was not of great semantic importance to the scene). Thus, we thought it likely that if no difference in detection time was found between categorical changes and metric changes, then a difference certainly would be found between the position changes and the presence/absence change.

\section{Method}

Participants. The participants were 60 college students. All participants reported normal or corrected-to-normal vision. They received course extra credit for participation.

Apparatus. Stimuli were presented on a 17-in. Apple color monitor with a resolution of $832 \times 624$ pixels and a vertical refresh rate of $75 \mathrm{~Hz}$. Stimuli were 15 realistic color scenes (designated standard scenes) of real-world environments created with 3-D graphics software. The number of objects (excluding background objects, such as walls and floors) in each scene ranged from 5 to 22 , with a
Standard Scene
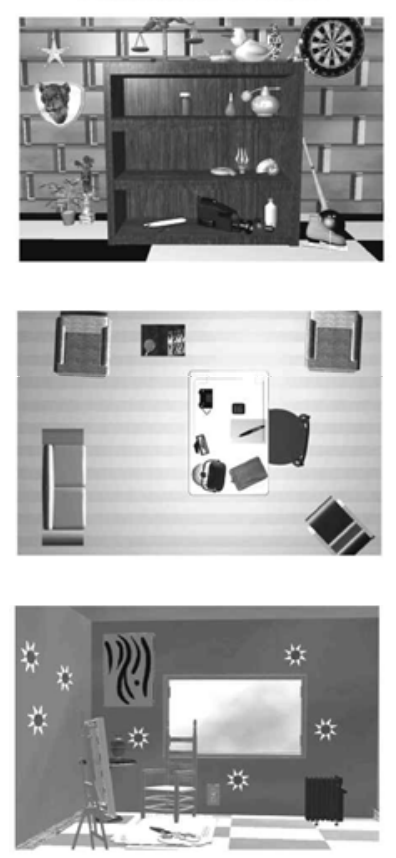

\section{Conditions}

\section{Categorical Change}
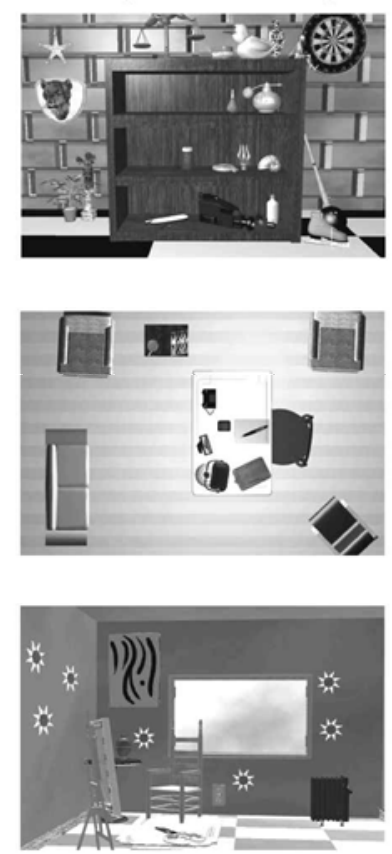

Metric Change
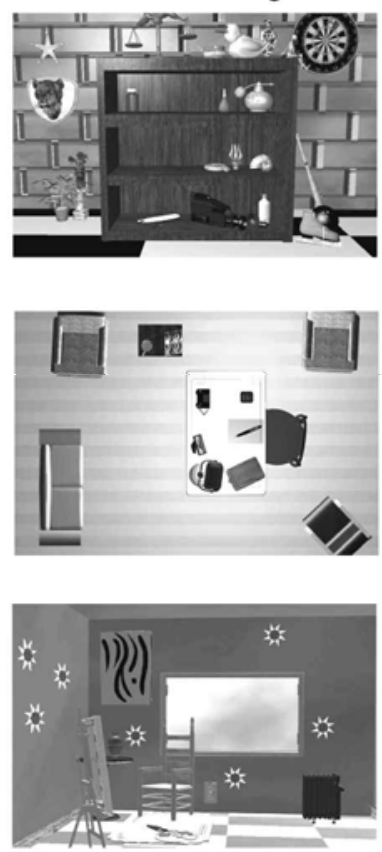

Presence / Absence
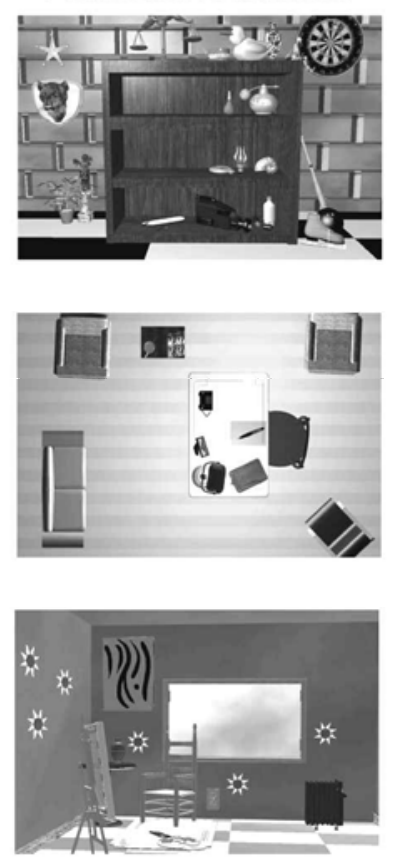

Figure 2. Illustration of the conditions and stimuli used in Experiment 1. On each trial, the participants viewed one of the standard scenes alternating with either the categorical changed version of the scene, the metric changed version of the scene, or the presence/absence version of the scene. In the book shelf scene (top row), the object that changes is the bottle on the top shelf in the standard scene. In the office scene (middle row), the object that changes is the calculator on the desk by the notepad. In the studio scene (bottom row), the object that changes is the star on the wall above the right-hand corner of the window. The original stimuli were in color. 
mean of 13. At the distance viewed by the participants in the experiments, each scene subtended $9.3^{\circ}$ horizontal $\times 6.3^{\circ}$ vertical of visual angle. The participants responded using a two-button response box attached to a National Instruments NB-DIO-24 board that gave \pm 0.5 -msec response time (RT) accuracy.

We created three versions of each standard scene by selecting one of the objects in each of the standard scenes and changing its position in one of three ways (Figure 2). In the presence/absence scenes, the object was simply deleted from the scene. In the categorical changed scenes, the object moved such that its categorical relationship to the closest object changed (e.g., went from above the closest object to the side of the closest object). In the metric changed scenes, the same object moved so that it did not change its categorical relationship to the closest object in the scene. For all position changes, the "closest object" excluded objects upon which the target object rested, such as the shelf in Figure 2, and excluded background objects such as walls, floor, and sky. Importantly, the distance moved by the object in the categorical changed version of a scene, and the metric changed scene version of a scene was identical. The object moved between $0.4^{\circ}$ and $1.3^{\circ}$ of visual angle, with a mean of $1.0^{\circ}$. In addition, objects moved only in the picture plane (and never in depth) so that the size of the object remained constant across position changes. None of the position changes involved moving an object behind or in front of another object, and none of the position changes produced unnatural arrangements of objects. For example, objects were not suspended in midair, and objects were not moved into unusual positions (e.g., a picture frame hanging on a wall was not moved to a position on a wall below an electrical outlet). Finally, care was taken so that both the metric change and the categorical change either did not disrupt the symmetry of a particular scene or did disrupt symmetry.

In each of the three versions of a scene, only one object was moved or deleted, and the same object was moved or deleted in each of the three versions. Thus, in a particular scene, the changed object was the same across all three conditions. The only difference was the type of change.

Also used in Experiment 1 was a mask. The mask was of the same dimensions as the scene stimuli $\left(9.3^{\circ}\right.$ horizontal $\times 6.3^{\circ}$ vertical of visual angle) and was composed of a random collection of object parts.

Procedure. The participants saw a fixation cue for $480 \mathrm{msec}$, followed by the standard version of a scene for $160 \mathrm{msec}$, followed by a mask for $160 \mathrm{msec}$, followed by one of the three altered versions of the standard scene for $160 \mathrm{msec}$, followed by the mask for $160 \mathrm{msec}$. The sequence of standard, mask, altered scene, mask repeated until the participant made a response or 40 repetitions had elapsed. The participants' responses were timed from initial presentation of the altered version of the scene (the second scene in the trial sequence).

The participant's task was to find the object that was changing between the two versions of the scene and to press the left button on a two-button response box when the change was detected (the right button on the response box was never used). The participant was required to immediately report the identity of the object (or describe the object if the participant was unsure of the object's identity). If the participant named an incorrect object or did not respond before the trial sequence terminated, then an error was counted.

Each of the 15 standard scenes was viewed by a participant once during the 15 experimental trials. On one third of the trials, the standard scene alternated with the categorical changed version of the scene (categorical change condition). On one third of the trials, the standard scene alternated with the metric changed version of the scene (metric change condition). On the remaining third of the trials, the standard scene alternated with the presence/absence version of the scene (presence/absence condition). The order of trials was random. Each alternative version of a scene (categorical, metric, or presence/absence) appeared equally often over the course of the experiment.

Prior to the experiment, each participant was read a standard set of directions. The participants were told in the directions that objects would either change location or appear and disappear but were not informed as to the specific types of location changes (i.e., categorical and metric) that would occur. The participants also completed one practice trial on which no data were collected. The scene used in the practice trial did not appear in any of the experimental trials, and each participant viewed the same practice trial scene. The practice scene involved a presence/absence change.

\section{Results and Discussion}

Mean RTs are shown in Figure 3. Mean error percentage was $1.4 \%$ for the categorical change condition, $3.3 \%$ for the metric change condition, and $1.3 \%$ for the presence/ absence condition.

An alpha level of .05 was used for all analyses. A oneway within-participants analysis of variance (ANOVA) conducted on the RT data, with type of change (categorical vs. metric vs. presence/absence) as the single independent variable, showed a reliable effect $[F(2,58)=3.9$, $\left.M S_{\mathrm{e}}=10,218,797, p<.02\right]$. Post hoc tests using the least

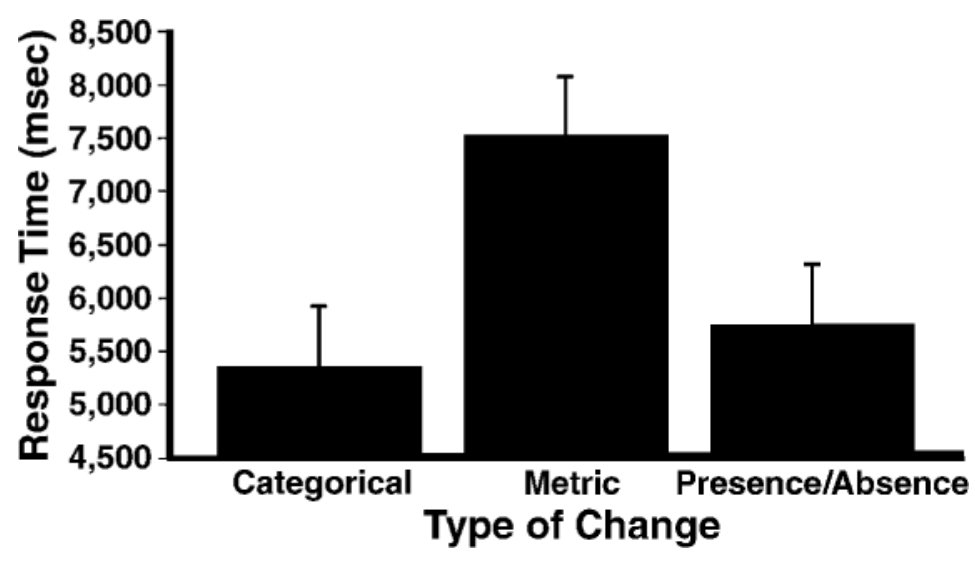

Figure 3. Mean response times for each condition in Experiment 1. Error bars represent the standard error of the mean (Loftus \& Loftus, 1988). 
significant difference (LSD) method indicated that the metric change condition produced reliably slower RTs than did the categorical change condition and the presence/absence condition ( $L S D=1,167 \mathrm{msec}$ ). No other pairwise comparisons were statistically reliable.

The participants found it more difficult to detect a change in the position of an object that did not disrupt its categorical relationship to the closest object in the scene than to detect an equal change to the same object that did disrupt its categorical relationship to the closest object in the scene. The results of Experiment 1 provide evidence that the categorical relations above, below, and side of are being coded in scene perception. In fact, the participants found it just as easy to detect categorical changes as they did to detect presence/absence changes. These results argue that categorical relations do play a role in coding the positions of objects in scenes.

In some of the scenes (e.g., the studio scene in Figure 2), the categorical changes appear to leave a region of empty space around the original location of the target object, whereas the metric changes do not appear to leave this region of empty space. A reviewer raised the possibility that the participants treated these categorical scenes like presence/absence scenes and thus were able to respond to the categorical version more quickly than to the metric version (leading to the categorical advantage).

Can a larger versus smaller region of empty space account for the results of Experiment 1? In order to test this hypothesis, an analysis of the entire stimulus set was conducted. The amount of empty space in a scene was measured as the mean distance from the target object to all the other objects in the scene (with object being defined using the same criteria outlined in the Method section). Thus, if a categorical change does leave this large area of open space, the categorical target object's location should be relatively close to other objects in the scene (because the objects in the scene should be clustered together, producing the region of empty space) compared with the metric object's location relative to other objects.

The amount of empty space was measured for the categorical and metric versions of each scene. Using this method of computing empty space, it was found that some of the categorical position changes produced a larger region of empty space than did the corresponding metric change ( 9 of the 15 scenes) and some of the categorical position changes produced a smaller region of empty space than did the corresponding metric change (6 of the 15 scenes).

If larger versus smaller regions of empty space were responsible for the results of Experiment 1, then the categorical advantage should be greater for scenes in which the categorical change produced more empty space (relative to the metric version) than for the scenes in which the categorical change produced less empty space (relative to the metric version). A between-participants $t$ test showed that this was not the case: There was no difference in the categorical advantage for scenes in which the categorical change produced a larger region of empty space and scenes in which the categorical change produced a smaller region of empty space $[t(13)=.07, S E=1,045$, n.s.]. Thus, we found no evidence that larger versus smaller regions of empty space were responsible for the results of Experiment 1 .

\section{EXPERIMENT 2}

The results of Experiment 1 demonstrated that changes that disrupt categorical relations are easier to detect than are equivalent changes that do not disrupt categorical relations. There are, however, at least two possible views of why this advantage to categorical changes in scene perception might occur. In both views, categorical changes are detected more quickly than are noncategorical changes because the two alternate versions of the scene that differ in their categorical relations would activate different memory representations of the scene. The views differ in whether the memory representations are preattentionally or attentionally produced and accessed. In a preattentional view, categorical changes might lead to the memory representations being compared unconsciously, leading to unconscious detection of the change. The unconsciously detected change would then direct attention to the location of the change, enabling conscious detection and overt reporting of the change. Thus, categorical relations might be coded and accessed preattentively and draw attention to the location of change when these relations are disrupted. In this view, categorical changes would result in a quicker allocation of attention to the location of the change than when only metric changes occur. An attentional view, in contrast, would posit that categorical relations might be coded and accessed only when spatial attention is focused on a local region of a scene. Thus, categorical changes would be detected only when spatial attention has already been allocated to the location of the change.

Some researchers have proposed that attentional processing is necessary for encoding categorical relations between parts of an object (Stankiewicz, Hummel, \& Cooper, 1998) and between multiple abstract objects (i.e., dashes and plus signs; see Logan, 1994). However, attention might not be required to encode the categorical relations between objects in a meaningful, contextual scene. There is evidence that participants can quickly determine the gist of a scene (e.g., Biederman, 1981), as well as report identities of objects in a scene even when the scene is processed under conditions of inattention (Mack \& Rock, 1998). Furthermore, participants quickly fixate the location of objects that do not fit into the overall meaning of a scene (the "octopus in a barnyard" effect of Loftus \& Mackworth, 1978). If the spatial relations between objects are part of the gist of a scene, it is possible that they are processed in a preattentional manner. Indeed, Rensink's (2000) coherence model of scene perception is ambivalent about the role of attention in the processing of spatial relations. According to the model, the coding of spatial layout (which 
presumably would include the categorical relations between the objects in a scene) is held in a nonattentional memory store.

To determine whether categorical relations in scene perception are computed preattentionally or attentionally, a $100 \%$ valid, central cue indicating the general spatial location of the object that would change was used in half of all trials in Experiment 2. In the attention literature, valid central cues result in quicker detection of targets, presumably because participants focus attention on the cued location (e.g., Jonides, 1981). Thus, on cued trials, attention should have been drawn to the location of the change. If categorical relations are encoded preattentionally and changes in categorical relations direct attention to the location of the change, directing attention to the location of the change with a cue (for both categorical and metric changes) should reduce the categorical advantage. That is, if the categorical advantage works by directing attention, directing attention in some other manner should reduce the advantage. If, however, categorical relations are encoded only after attention has been allocated to the location of the scene in which the change occurs, directing attention to the location of the change with a cue should have no effect on the categorical advantage. Providing the cue should have a main effect of decreasing the amount of time to detect the change for both categorical and metric change conditions. But if categorical relations are only encoded after attention is moved to a region of space, there should be no effect of the cue on the difference between categorical and metric change conditions. Thus, a preattentional view would predict an interaction between cue condition (cued or not cued) and type of change (metric or categorical), with a reduced effect of type of change when the scene is preceded by a cue. An attentional view would only predict main effects of cue condition and type of change.

\section{Method}

The participants were 80 students from the same subject pool as Experiment 1 . The same 15 scenes from Experiment 1 were used, plus an additional scene that was not used in Experiment 1. Only categorical and metric changed scenes were used (i.e., the presence/absence condition was not included). On half of the 16 trials ( 4 categorical change trials and 4 metric change trials), the initial presentation of the standard scene was preceded by a 1,920-msec presentation of a $100 \%$ valid cue that indicated the quadrant of the scene in which the changing object would appear. The cue consisted of a black screen with one quadrant in white. The participants were instructed that the white portion of the cue always indicated the area of the scene in which the change would occur. Each scene appeared in each condition an equal number of times over participants.

Prior to the experiment, each participant was read a standard set of directions and completed one practice trial on which no data were collected. The scene used in the practice trial did not appear on any of the experimental trials, and each participant viewed the same practice trial scene. The practice scene involved a cued, metric change. All other aspects of Experiment 2 were identical to those of Experiment 1.

\section{Results and Discussion}

The results can be seen in Figure 4. A within-participants factorial ANOVA was conducted on the RT data, with cue condition (cue or no cue) and type of change (categorical or metric) as independent variables. A reliable main effect was found for cue condition $\left[F(1,316)=162.25, M S_{\mathrm{e}}=\right.$ $8,209,919, p<.001]$, with faster RTs when the scene was preceded by a cue than when it was not. In addition, a reliable main effect was found for type of change $[F(1,316)=$ $\left.10.67, M S_{\mathrm{e}}=8,209,919, p<.005\right]$, with categorical changes leading to faster RTs than metric changes. The cue condition $\times$ type of change interaction, however, was not reliable $(F<1)$.

Experiment 2 replicated the pattern from Experiment 1, suggesting that categorical relations are coded in scene

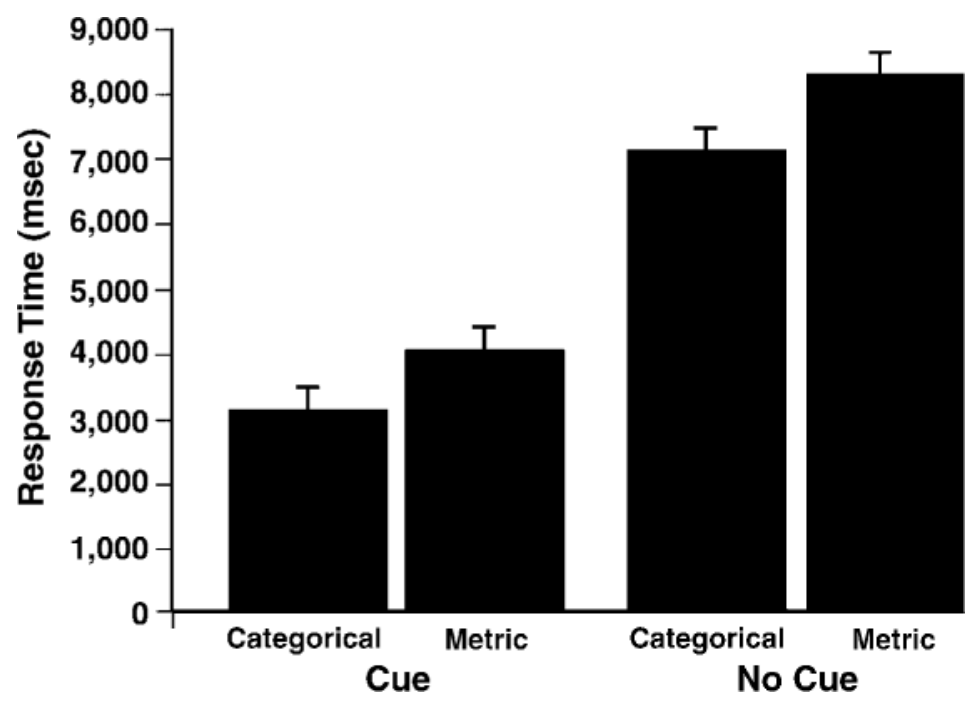

Figure 4. Mean response times for each condition in Experiment 2. Error bars represent the standard error of the mean. 
perception. Categorical changes were more easily detected than were metric changes. The size of the categorical advantage was not as great as it was in Experiment 1 . This reduction in the size of the categorical advantage suggests that the magnitude of the advantage is sensitive to the context in which the task occurs. The presence/ absence condition was used in Experiment 1, but not in Experiment 2. Cues were used in Experiment 2, but not in Experiment 1 . It is possible that these differences could have caused the participants to use different strategies in the two experiments, which might have resulted in the lowered categorical advantage in Experiment 2. Although the reduction in the advantage is interesting and will be investigated in future research, it does not detract from the major finding in Experiment 2. The participants were able to detect changes more quickly when the quadrant in which the change occurred was cued, but the difference between categorical and metric changes was not affected by the presence of a cue. Thus, Experiment 2 provides evidence that the coding of categorical relations is accomplished only after spatial attention has been allocated to a particular local area of a scene.

\section{GENERAL DISCUSSION}

The results of the present study provide evidence that categorical relations between objects are coded in scene perception (Experiments 1 and 2). Changes in scenes that disrupted above, below, or side of relations between two objects were more easily detected than were equivalent distance changes that did not disrupt these relations. Furthermore, the coding of categorical relations between objects appears to occur only when spatial attention has been allocated to the local area of the scene (Experiment 2). The level of the categorical advantage was not affected by the presence of a cue.

The present results suggest important parallels between object recognition and scene perception. Some models of object recognition (e.g., Hummel \& Biederman, 1992; Hummel \& Stankiewicz, 1996) posit that the parts of an object and the categorical relations among those parts are represented independently of each other. Attention serves to bind categorical relations together with object parts in order to form a complete representation of the object. The present results suggest that the same type of binding may be occurring in scene perception as well. Attention to a local area of a scene may bind objects and their categorical position together, forming a representation sufficiently detailed to enable the detection of change. Although Rensink's (2000) model of scene perception posits that only a single object is stabilized in attention at any one time, the present results suggest that multiple objects and their categorical relations might be stabilized and bound at once, in a manner similar to the multiple parts and their relations in a single object.

The results of Experiments 1 and 2 are some of the first to indicate categorical coding of relations in scene perception. Several recent studies have found evidence that the spatial relations between multiple objects are coded only metrically (e.g., Diwadkar \& McNamara, 1997; Shelton \& McNamara, 1997), but these studies either employed tasks that explicitly encouraged metric coding (e.g., Shelton \& McNamara, 1997, had participants point to the location of an object) or used abstract stimuli such as disconnected object parts or line segments (e.g., Humphreys \& Riddoch, 1995). The relations between multiple abstract objects and the relations between objects in three-dimensional scenes (such as the scenes employed in the present study) may be treated differently by the visual system.

However, the results of Experiments 1 and 2 do not imply that metric information is not coded in scene perception; in fact, the results support the idea that metric information is being coded. If no metric information were included, then the participants would have found the metric-only changes virtually impossible to detect. Rather, position in scene perception might be coded using a nonlinear output function that includes both categorical information and metric information (see Hummel \& Stankiewicz, 1998). Alternatively, both categorical information and metric representations of object locations may be coded independently of each other, but both types of representation may be used to guide change detection.

Although the results of the present study show a categorical advantage for detecting changes to scenes, they do not speak to why this advantage exists. It could be the case that people attend to regions of a display along categorical lines (e.g., by first attending above a selected object in a display, then side of the object), thus allowing for faster detection of categorical disruptions. Alternatively, categorical relations may simply be extracted more quickly under attention than may metric relations (e.g., Kosslyn, Chabris, Marsolek, \& Koenig, 1992), allowing for faster detection of categorical changes. Likewise, the results do not speak to whether the attentional costs (as measured by invalid cuing) for change detection would be different for categorical and metric changes. The answers to both of these questions will help further our understanding of the role of categorical relations and attention in scene perception.

After attention leaves the local area of the scene, the fate of the representation containing the objects and their categorical relations is unclear. It is possible that the categorical relations between objects are coded only when focused attention is allocated to the local area of a scene and are lost when focused attention leaves the local area of the scene. Such a view would be in agreement with views of visual search that posit preattentional and postattentional processing of objects are equivalently degraded (e.g., Horowitz \& Wolfe, 1998). Alternatively, the categorical relations between objects in scenes could be attentionally encoded into a long-term memory representation that can then be retrieved when focused attention returns to the same local area (e.g., Henderson \& Hollingworth, $1999 b)$. Note, however, that in both views categorical relations are coded. Furthermore, the initial encoding of the categorical relations can be accomplished only through attentional processing and can be "used" only when atten- 
tion is focused on the local area of the scene. Future research will be needed to discriminate between these two alternative views.

Likewise, future research will also need to specify more precisely the representation used for scene perception. Although we found evidence that above, below, and side of are being coded relative to the closest object in a scene, it is possible that these relations are coded relative to other objects as well, perhaps all other objects in foveal vision. It is also possible that relations other than above, below, and side of are being coded, such as the relation between. The flicker paradigm can provide a useful tool for investigating these questions.

\section{REFERENCES}

Archambault, A., O'Donnell, C., \& Schynes, P. G. (1999). Blind to object changes: When learning the same object at different levels of categorization modifies its perception. Psychological Science, 10, 249-255.

Biederman, I. (1981). On the semantics of a glance at a scene. In M. Kubovy \& J. R. Pomerantz (Eds.), Perceptual organization (pp. 213-253). Hillsdale, NJ: Erlbaum.

Biederman, I. (1987). Recognition-by-components: A theory of human image understanding. Psychological Review, 94, 115-147.

Biederman, I., \& Gerhardstein, P. C. (1995). Viewpoint-dependent mechanisms in visual object recognition: Reply to Tarr and Bülthoff (1995). Journal of Experimental Psychology: Human Perception \& Performance, 21, 1506-1514.

Bülthoff, H. H., \& Edelman S. (1992). Psychophysical support for a two-dimensional view interpolation theory of object recognition. Proceedings of the National Academy of Science, 89, 60-64.

Carlson-Radvansky, L., \& IRwin, D. E. (1995). Memory for structural descriptions across eye movements. Journal of Experimental Psychology: Learning, Memory, \& Cognition, 21, 1441-1458.

Cooper E. E., \& Wojan, T. J. (2000). Differences in the coding of spatial relations in face identification and basic level object recognition. Journal of Experimental Psychology: Learning, Memory, \& Cognition, 26, 470-488.

Diwadkar, V. A., \& McNamara, T. P. (1997). Viewpoint dependence in scene recognition. Psychological Science, 8, 302-307.

EPSTEIN, R, \& KANWISHER, N. (1998). A cortical representation of the local visual environment. Nature, 392, 598-601.

Henderson, J. M., \& Hollingworth, A. (1999a). High-level scene perception. Annual Review of Psychology, 50, 243-271.

Henderson, J. M., \& Hollingworth, A. (1999b). The role of fixation position in detecting scene changes across saccades. Psychological Science, 10, 438-443.

Horowitz, T. S., \& WolfE, J. M. (1998). Visual search has no memory. Nature, 394, 575-577.

Hummel, J. E., \& Biederman, I. (1992). Dynamic binding in a neural network for shape recognition. Psychological Review, 99, 480-517.

Hummel, J. E., \& Stankiewicz, B. J. (1996). Categorical relations in shape perception. Spatial Vision, 10, 201-236.

Hummel, J. E., \& Stankiewicz, B. J. (1998). Two roles for attention in shape perception: A structural description model of visual scrutiny. Spatial Vision, 5, 49-79.

Humphreys, G. W., \& RidDoch, M. J. (1994). Attention to withinobject and between-object spatial representations: Multiple sites for visual selection. Cognitive Neuropsychology, 11, 207-241.

Humphreys, G. W., \& RidDoch, M. J. (1995). Separate coding of space within and between perceptual objects: Evidence from unilateral visual neglect. Cognitive Neuropsychology, 12, 283-311.

IRWIN, D. E., \& ANDREWs, R. V. (1996). Integration and accumulation of information across saccadic eye movements. In T. Inui \& J. McClelland (Eds.), Attention and performance XVI (pp. 125-156). Cambridge, MA: MIT Press.

JoNIDES, J. (1981). Voluntary versus automatic control over the mind's eye's movement. In J. Long \& A. D. Baddeley (Eds.), Attention and performance IX (pp. 187-203). Hillsdale, NJ: Erlbaum.

Kosslyn, S. M., Chabris, C. F., Marsolek, C. J., \& Koenig, O. (1992). Categorical versus coordinate spatial relations: Computational analysis and computer simulations. Journal of Experimental Psychology: Human Perception \& Performance, 18, 562-577.

LeVIn, D. T., \& Simons, D. J. (1997). Failure to detect changes to attended objects in motion pictures. Psychonomic Bulletin \& Review, 4, 501-506.

Loftus, G. R., \& Loftus, E. F. (1988). Essence of statistics (2nd ed.). New York: Random House.

Loftus, G. R., \& MACKworth, N. H. (1978). Cognitive determinants of fixation location during picture viewing. Journal of Experimental Psychology: Human Perception \& Performance, 4, 565-572.

Logan, G. D. (1994). Spatial attention and the apprehension of spatial relations. Journal of Experimental Psychology: Human Perception \& Performance, 20, 1015-1036.

MAck, A., \& Rock, I. (1998). Inattentionalblindness. Cambridge, MA: MIT Press.

Rensink, R. A. (2000). The dynamic representation of scenes. Visual Cognition, 7, 17-42.

Rensink, R. A., O'Regan, J. K., \& Clark, J. J. (1997). To see or not to see: The need for attention to perceive changes in scenes. Psychological Science, 8, 368-373.

Rensink, R. A., O'Regan, J. K., \& Clark, J. J. (2000). On the failure to detect changes in scenes across brief interruptions. Visual Cognition, 7, 127-145.

Rosielle, L. J., \& Cooper, E. E. (2001). Categorical perception of relative orientation in visual object recognition. Memory \& Cognition, 29, 68-82.

SAiki, J., \& Hummel, J. E. (1998a). Connectedness and the integration of parts with relations in shape perception. Journal of Experimental Psychology: Human Perception \& Performance, 24, 227-251.

SAIKI, J., \& Hummel, J. E. (1998b). Connectedness and part-relation integration in shape category learning. Memory \& Cognition, 26, 1138-1156.

Shelton, A. L., \& McNamara, T. P. (1997). Multiple views of spatial memory. Psychonomic Bulletin \& Review, 4, 102-106.

Simons, D. J., \& WANG, F. (1998). Perceiving real-world viewpoint changes. Psychological Science, 9, 315-320.

Stankiewicz, B. J., Hummel, J. E., \& Cooper, E. E. (1998). The role of attention in priming for left-right reflections of object images: Evidence for a dual representation of shape. Journal of Experimental Psychology: Human Perception \& Performance, 24, 732-744.

TARR, M. J., \& BüLThOFF, H. H. (1995). Is human object recognition better described by geon structural descriptions of by multiple views? Comment on Biederman and Gerhardstein (1993). Journal of Experimental Psychology: Human Perception \& Performance, 21, 14941505.

TARr, M. J., \& PINker, S. (1990). When does human object recognition use a viewer-centered reference frame? Psychological Science, 1, 253256.

TARr, M. J., \& PINKer, S. (1991). Orientation-dependent mechanisms in shape recognition: Further issues. Psychological Science, 2, 207-209.

Ullman, S. (1989). Aligning pictorial descriptions: An approach to object recognition. Cognition, 32, 193-254.

Ullman, S. (1996). High-level vision: Object recognition and visual cognition. Cambridge, MA: MIT Press.

WANG, F., \& Simons, D. J. (1999). Active and passive scene recognition across views. Cognition, 70, 191-210.

(Manuscript received April 19, 2000; revision accepted for publication November 20, 2000.) 